\title{
Adaptive model to apply the Upper-Bound Theorem in plain strain forging
}

\author{
F. Martín ${ }^{1, a}$, L. Sevilla ${ }^{1, b}$ A. Camacho ${ }^{2, c}$ and A. Sanz ${ }^{3, d}$ \\ ${ }^{1}$ Department of Manufacturing Engineering. University of Malaga. Malaga. Spain \\ ${ }^{2}$ Department of Manufacturing Engineering. National Distance University of Spain. Madrid. Spain \\ ${ }^{3}$ Department of Aerospace Materials and Production. Technical University of Madrid. Madrid, Spain \\ afdmartin@uma.es, 'Isevilla@uma.es, camcamacho@ind.uned.es, da.slobera@upm.es
}

Keywords: Metal forming, Forging, Upper-Bound Theorem, Friction, Analytical methods

\begin{abstract}
Present work applies the Upper-Bound Theorem (UBT) with Triangular Rigid Blocks (TRB) to metal forming compression processes like plane strain forge, offering an upper limit to required deformation energy. This analytical method, usually used by means of simplified models, is developed here incorporating different effects that impact in evolution of deformation process like shape factor and friction. By means of a new adaptive model, the shape and size of the rigid zones used for the UBT application are optimized according to the ratio of the width and the height of workpiece.
\end{abstract}

\section{Introduction}

Modern, exact solutions in metal forming processes are very difficult to obtain, due to their high degree of complexity on involved parameters. However, according to the bound theorems, it is possible to make a quite accurate approximation by confining the solution of necessary deformation energy between upper and lower limits. The Upper-Bound Theorem (UBT) is an analytical method that allows obtaining enough admissible solutions using a separation of a deformed workpiece in rigid zones, called Triangular Rigid Blocks (TRB) in plain strain problems [1]. Modern trends in this field tried to extend the application of this analytical method to more general situations [2-4]. In this work, an analysis of a new adaptive model is developed to improve the UBT application in forging processes. This procedure is based in a model with a flexible TRB configuration $[5,6]$ and allows to improve the solutions obtained by classical UBT procedures by comparing the results obtained with different configurations, according to the "shape factor", that is the ratio of the width and the height of the workpiece section to be analyzed.

\section{Methodology}

A prismatic workpiece with an initial $2 h$ height, $2 b$ width and $w$ depth has been considered as a geometrical model. Due to the double symmetry, only a quarter of the billet is necessary to be considered (Fig. 1).

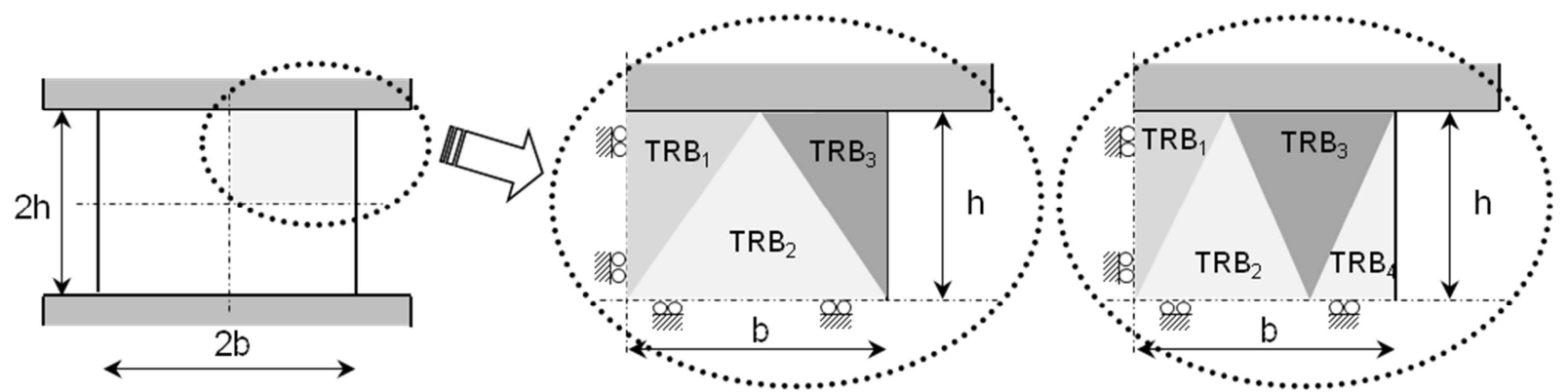

Figure 1. Shape of the workpiece and examples of distributions with 3 or 4 TRB 
The geometrical parameters of each TRBs configuration and their hodograph are represented in Fig. 3 and Fig.4 for an odd and an even number of TRBs [7] to study the influence of the TRB number in the $p / 2 k$ relation, where $p$ is the mean forging pressure and $k$ is the shear yield stress of this material.
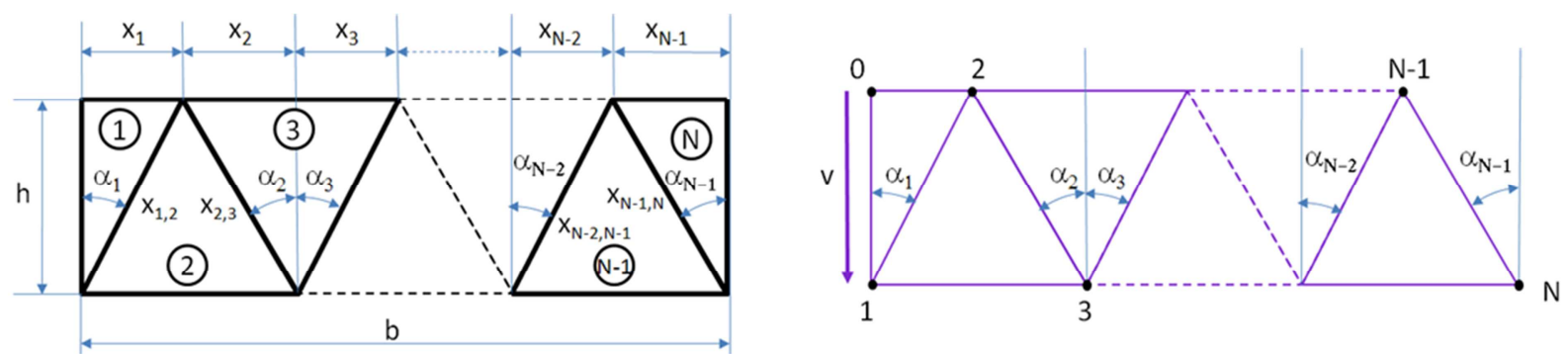

Figure 2. Distribution and hodograph with odd number of TRB
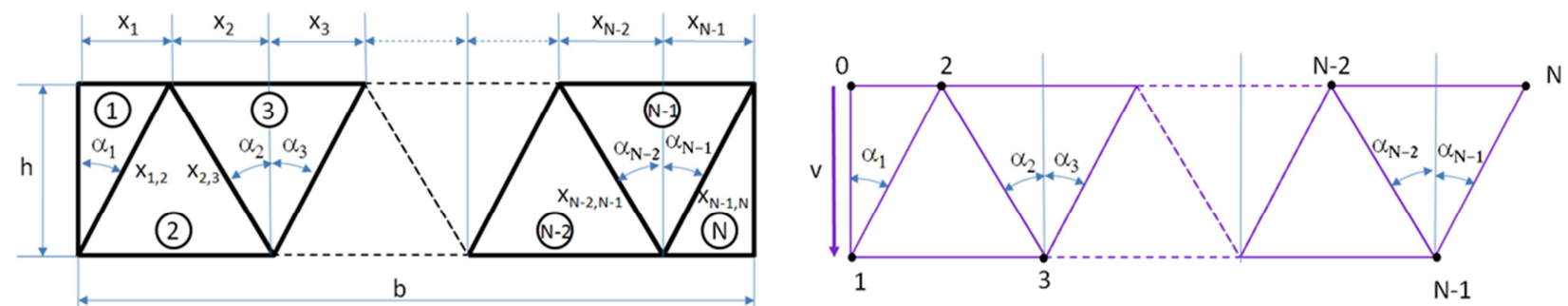

Figure 3. Distribution and hodograph with even number of TRB

The application of the UBT (Eq. 1) in each of these four cases should obtain optimized values of $x_{i}$ parameters in different boundary conditions [8].

$$
\int_{\mathrm{S}_{\mathrm{V}}} \mathrm{T}_{\mathrm{i}} \mathrm{v}_{\mathrm{i}} \mathrm{dS} \mathrm{S}_{\mathrm{V}} \leq \int_{\mathrm{S}_{\mathrm{D}}^{*}} \mathrm{k}\left[v^{*}\right] \mathrm{dS}_{\mathrm{D}}^{*}+\int_{\mathrm{S}_{\mathrm{F}}} \mathrm{T}_{\mathrm{i}} \mathrm{v}_{\mathrm{i}}^{*} \mathrm{~d} \mathrm{~S}_{\mathrm{F}}
$$

Where $T_{i}$ : external surface stresses on the workpiece to form; $v_{i}$ : actual velocity field; $S_{v}$ : surfaces where the external forces are applied; $k$ : shear yield stress; $v^{*}$ : velocity discontinuities; $S^{*}{ }_{D}$ : discontinuity surfaces; $v_{i}^{*}$ : kinematically admissible virtual velocity field; $S_{F}$ : external surfaces exposed to external surface stresses.

The consideration of a semi-adherence in die-workpiece contact has been solved including an $m$ friction shear factor in affected terms of Eq. $1[5,8]$.

The $p / 2 k$ relation is evaluated according to shape factor over a variable number of TRB, obtaining a generalization for $n$ TRB expression. Eq. 2 and Eq. 3 show the deformation power expressions for an odd number (Eq.2) and an even number (Eq.3) of TRBs:

$$
\begin{aligned}
& \frac{d W}{d t}=\dot{W} \leq k w\left[\sum_{i=1}^{N-1} v_{i, i+1} x_{i, i+1}+m \sum_{i=1}^{(N-1) / 2} v_{1,2 i+1}\left(x_{2 i}+x_{2 i+1}\right)\right] ; \quad x_{N}=0 \\
& \frac{d W}{d t}=\dot{W} \leq k w\left[\sum_{i=1}^{N-1} v_{i, i+1} x_{i, i+1}+m \sum_{i=1}^{N / 2-1} v_{1,2 i+1}\left(x_{2 i}+x_{2 i+1}\right)\right]
\end{aligned}
$$

Applying the trigonometric ratios for the positions and velocities of each block is possible to simplify these equations, obtaining Eq. 4 for an odd number of TRBs

$$
\frac{d W}{d t}=\dot{W} \leq \frac{k w v}{h}\left[h^{2}(N-1)+\sum_{i=1}^{N-1} x_{i}^{2}+m \sum_{i=1}^{(N-1) / 2}\left(\sum_{j=1}^{2 i} x_{j}\right)\left(x_{2 i}+x_{2 i+1}\right)\right] ; \quad x_{N}=0
$$

and Eq. 5 for an even number of TRBs.

$$
\frac{d W}{d t}=\dot{W} \leq \frac{k w v}{h}\left[h^{2}(N-1)+\sum_{i=1}^{N-1} x_{i}^{2}+m \sum_{i=1}^{(N-2) / 2}\left(\sum_{j=1}^{2 i} x_{j}\right)\left(x_{2 i}+x_{2 i+1}\right)\right]
$$


Differentiating with respect to each xi and equating to zero, the values of xi that minimize the power of deformation process are obtained, defining geometrical configuration of different TRB combinations (Eq. 6 and Eq.7)

$$
x_{i}=\frac{b}{2(N-1)}\left(2+(-1)^{i+1} m\right) \quad \forall i=\{1, . ., N-1\}
$$

for an odd number of TRBs and

$$
x_{i}=\frac{b}{2(N-1)+m}\left(2+(-1)^{i+1} m\right) \quad \forall i=\{1, . ., N-1\}
$$

for an even number of TRBs.

\section{Results}

With precedent expressions the value of parameters to define the geometry of TRB in a concrete configuration is obtained. Different $p / 2 k$ relations have been calculated applying these equations and selecting the initial velocity $v$ as unity value and the $m$ friction factor between 0 and 1 values.

The obtained values for friction equal to zero have been compared with Johnson-Mellor expression, Eq. 8 [7], being extremely concordant (Fig. 4). In this equation, the authors select discontinuity lines with equal angles with respect to horizontal central plane of the deformation body.

$$
\frac{p}{2 k}=\frac{1}{2}\left(\frac{b / h}{n}+\frac{n}{b / h}\right)
$$

Where $n$ is the number of intersections of the discontinuity over the upper surface (Fig.2) and $b / h$ is the shape factor.
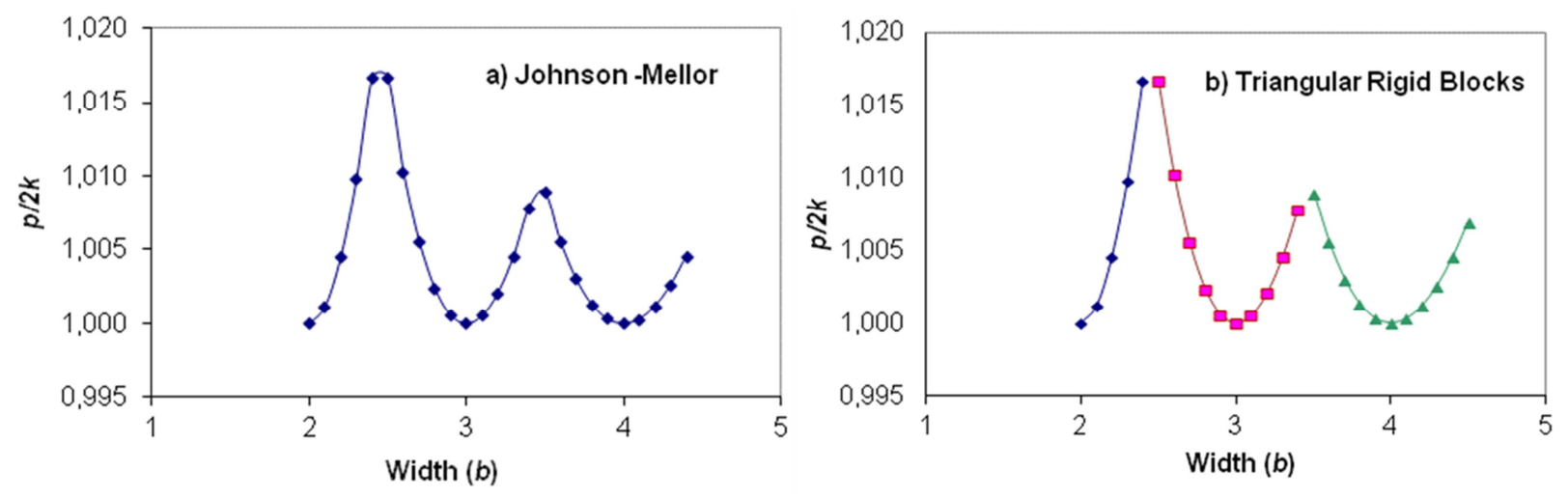

Figure 4. a) Johnson-Mellor results. b) UBT-TRB results

Finally, Fig. 5 shows the values of the $p / 2 k$ dimensionless relation with different $b / h$ shape factor, $n$ number of TRB and $m$ friction factor. The maximum shape factor considered is 6 , which is considered a limit for technologically viable deformations. It allows selecting the best solution depending on process conditions and to adapter the shape and size of the rigid zones. 

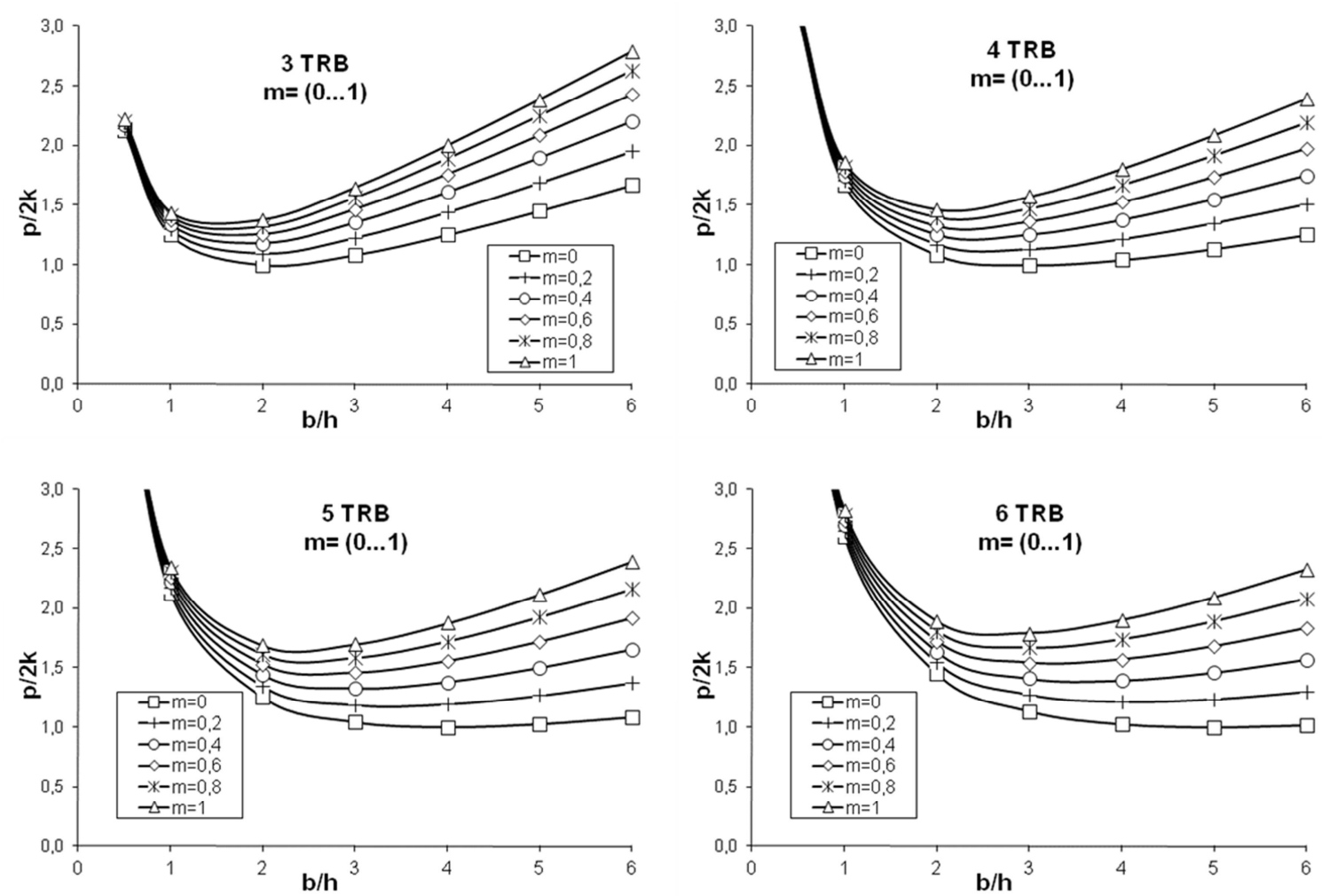

Figure 5. $p / 2 k$ values versus $b / h$ for $3,4,5$ and 6 TRB and different friction ratios

\section{Conclusion}

In the present work, forging processes have been analyzed under plane strain conditions in different conditions of the workpiece reduction. The evolution of $p / 2 k$ has been obtained by means of the Upper Bound Theorem. The results of the developed model have been contrasted with Johnson \& Mellor expression with a very good concordance. Four distributions of TRB have been considered to analyze the influence of the friction and the shape factor. The shape and size of the rigid zones are optimized according to the ratio of the width and the height of workpiece by an adaptive model. This method covers a wide range of common geometric configurations of industrial forged products.

\section{Acknowledgements}

This work was financially supported by the Ministry of Economy and Competitiveness of Spain (Project DPI2009-07300).

\section{References}

[1] F. Martin, L. Sevilla and C. Bermudo: Mater. Sci. Forum Vol. 713 (2012), p. 13

[2] I.A. Kan, V Bhasin, et al: Int. J. Solids Struct. Vol. 45 (25-26) (2008), p. 6416

[3] V. Ranatunga and J. Gunasekera: J. Mater. Eng. Perform. Vol. 15 (1) (2006), p. 47

[4] K. Abrinia and A. Fazlirad: J. Mater. Process. Technol. Vol. 209 (7) (2009), p. 3264

[5] E.M. Rubio, A.M. Camacho, et al.: Mater. Manuf. Process. Vol. 23 (7) (2008), p. 690

[6] E.M. Rubio, M. Marin, et al.: Int. J. Adv. Manuf. Technol. Vol. 40 (3-4) (2009), p. 261

[7] W. Johnson and P.B. Mellor: Engineering Plasticity (Ellis Horwood Limited, New York 1983)

[8] J. Chakrabarty: Theory of Plasticity (Elsevier Butterworth-Heinemann, Oxford 2006) 